\title{
Analysis of differences in the stability of proteins encoded in mitochondrial DNA of model organisms
}

\author{
KRZYSZTOF KOWAL, ADAM BOWNIK, ANGELIKA TKACZYK, BRYGIDA ŚLASKA \\ Institute of Biological Bases of Animal Production, Faculty of Biology, Animal Sciences and Bioeconomy, \\ University of Life Sciences in Lublin, Akademicka 13, 20-950 Lublin, Poland
}

Kowal K., Bownik A., Tkaczyk A., Ślaska B.

Analysis of differences in the stability of proteins encoded in mitochondrial DNA of model organisms Summary

The aim of this study was to describe the stability of proteins encoded in mtDNA, which are part of the OXPHOS system, in different model organisms and to define why certain proteins are more prone to be unstable than others. The in silico analyses involved 155 reference sequences of all proteins encoded in the mitochondrial DNA in twelve model organisms representing different phylogenetic groups. The amino acid sequences of the proteins were taken from the GenPept database. The bioinformatic analyses were performed in the ProtParam program. Thirty-eight of the $\mathbf{1 5 5}$ analyzed proteins exhibited instability. The greatest numbers of unstable mitochondrial proteins were detected in $\mathrm{H}$. sapiens and $A$. mexicanum and the lowest levels were found in C.elegans. ND1 and ATP8 were the most unstable mitochondrial proteins. Proteins COX1 and COX3 did not exhibit instability in the examined group of organisms. The highest instability index values were recorded in the case of protein ATP8. Protein ND1 turned out to be stable in the representatives of the class invertebrates. The preliminary results of the pioneer investigations indicate that the type and number of unstable proteins encoded in mtDNA was species specific. Protein instability in lower organisms may be associated with resistance to oxidative stress. In higher organisms, in turn, protein instability may be related to the physiological production of free oxygen radicals, which play multiple roles in metabolic processes. The phenomenon of instability in the respiratory chain proteins may have a strategic function although it appears to be detrimental to the stability of the protein structure per se.

Keywords: instability index, mitochondrial proteins, reactive oxygen species

The genetic information on the structure of 37 genes, i.e. 13 respiratory chain proteins, 22 tRNA molecules, and 2 rRNA molecules, is encoded in the mitochondrial genome. Mitochondrial DNA (mtDNA) contains a non-coding region (D-loop), whose length varies among animals. Due to the lack of introns, genes in some organisms overlap, e.g. in humans, the ATP6 and ATP8 genes share 46 nucleotides and the ND4 and ND4L genes share seven nucleotides $(2,8)$. The length of mtDNA is highly variable in different organisms, ranging from yeasts to higher eukaryotes. However, certain mtDNA properties are conserved, e.g. the presence of genes coding for highly hydrophobic and crucial components of the respiratory chain, ribosomal RNAs (rRNAs), and transfer RNAs (tRNAs), which are necessary for protein translation (5). How electron transfer is coupled to proton pumping is a major unresolved question (19). Many metabolic processes, e.g. the Krebs cycle, the urea cycle, or $\beta$-oxidation, take place in the mitochondria. Additionally, mitochondria are involved in the regulation of thermogenesis and $\mathrm{Ca}^{2+}$ ion metabolism and exert an effect on the apoptosis process. However, the main role of mitochondria is generation of high-energy ATP in the process of oxidative phosphorylation (9). The respiratory chain consists of 5 protein complexes: NADH-ubiquinone oxidoreductase, succinate-quinone oxidoreductase, the $b c l$ complex, cyt c oxidase, and ATP synthase. The complexes are arranged on the internal mitochondrial membrane according to the increasing oxidoreductive potential. The difference in the potentials between the first four complexes facilitates electron transport. It results in generation of energy, which is converted by ATP synthase into high-energy phosphorus bonds (14).

Approximately $0.15-2 \%$ of electrons that enter the electron transport chain (ETC) can escape the OXPHOS cycle, resulting in the formation of superoxide (22). Increased exposure to this OXPHOS-related superoxide may affect not only the mtDNA located nearby, but also proteins and lipids, resulting in impaired protein 
Tab. 1. List of reference sequences used in the analysis of the stability of mitochondrial proteins

\begin{tabular}{|c|c|c|c|c|c|c|c|}
\hline $\begin{array}{c}\text { Organism } \\
\text { /Protein }\end{array}$ & $\begin{array}{c}\text { Caenorhabditis } \\
\text { elegans }\end{array}$ & Daphnia magna & $\begin{array}{c}\text { Drosophila } \\
\text { melanogaster }\end{array}$ & $\begin{array}{c}\text { Latimeria } \\
\text { chalumna }\end{array}$ & Danio rerio & $\begin{array}{c}\text { Ambystoma } \\
\text { mexicanum }\end{array}$ & $\begin{array}{c}\text { Crocodylus } \\
\text { porosus }\end{array}$ \\
\hline ATP6 & NP_006956.1 & YP_009133118.1 & YP_009047270.1 & NP_008334.1 & NP_059336.1 & NP_990981.1 & YP_637145.1 \\
ATP8 & N/D* & YP_009133117.1 & YP_009047269.1 & NP_008333.1 & NP_059335.1 & NP_990980.1 & YP_637144.1 \\
C0X1 & NP_006961.1 & YP_009133115.1 & YP_009047267.1 & NP_008331.1 & NP_059333.1 & NP_990978.1 & YP_637142.1 \\
C0X2 & NP_006962.1 & YP_009133116.1 & YP_009047268.1 & NP_008332.1 & NP_059334.1 & NP_990979.1 & YP_637143.1 \\
C0X3 & NP_006959.1 & YP_009133119.1 & YP_009047271.1 & NP_008335.1 & NP_059337.1 & NP_990982.1 & YP_637146.1 \\
\hline CYTB & NP_006958.1 & YP_009133125.1 & YP_009047277.1 & NP_008341.1 & NP_059343.1 & NP_990988.1 & YP_637152.1 \\
\hline ND1 & NP_006955.1 & YP_009133126.1 & YP_009047278.1 & NP_008329.1 & NP_059331.1 & NP_990976.1 & YP_637140.1 \\
\hline ND2 & NP_006957.1 & YP_009133114.1 & YP_009047266.1 & NP_008330.1 & NP_059332.1 & NP_990977.1 & YP_637141.1 \\
\hline ND3 & NP_006963.1 & YP_009133120.1 & YP_009047272.1 & NP_008336.1 & NP_059338.1 & NP_990983.1 & YP_637147.1 \\
\hline ND4 & NP_006960.1 & YP_009133122.1 & YP_009047274.1 & NP_008338.1 & NP_059340.1 & NP_990985.1 & YP_637149.1 \\
\hline ND4L & NP_006954.1 & YP_009133123.1 & YP_009047275.1 & NP_008337.1 & NP_059339.1 & NP_990984.1 & YP_637148.1 \\
\hline ND5 & NP_006964.1 & YP_009133121.1 & YP_009047273.1 & NP_008339.1 & NP_059341.1 & NP_990986.1 & YP_637150.1 \\
\hline ND6 & NP_006953.1 & YP_009133124.1 & YP_009047276.1 & NP_008340.1 & NP_059342.1 & NP_990987.1 & YP_637151.1 \\
\hline
\end{tabular}

Explanations: ${ }^{*}$ C. elegans does not contain a gene encoding the ATP8 protein

synthesis and/or enhanced reactive oxygen species (ROS) production (20). ROS is a collective term which includes superoxide, hydrogen peroxide, and hydroxyl free radicals. The main enzyme responsible for generation of the ROS complex is NADH-ubiquinone oxidoreductase, which is the largest enzyme of the OXPHOS system formed by 44 subunits, from which seven are encoded by mtDNA (12).

The instability index provides an estimate of the stability of a protein in a test tube. As shown by Guruprasad et al. (10), there are certain dipeptides whose occurrence is significantly different in unstable proteins compared with those present in stable ones. The authors of this method have assigned a weight value of instability to each of the 400 different dipeptides. With these weight values, it is possible to compute an instability index (10). The authors have also indicated that point mutations may alter the stability of a protein.

To date, there is no information in the literature on the occurrence of unstable mitochondrial proteins in various organisms. There is also no answer to the question of the physiological presence of unstable proteins in the cell. Therefore, these preliminary studies were undertaken to attempt at clarification of these issues. The inclusion of all proteins encoded in mitochondrial DNA in representatives of different groups of phylogenetic organisms may be the first step in elucidation of the presence of unstable mitochondrial proteins in physiological conditions.

The aim of this study was to describe the stability of proteins encoded in mtDNA, which are part of the OXPHOS system, in different model organisms and to define why certain proteins are more prone to be unstable than the others.

\section{Material and methods}

The in silico analyses involved 155 reference sequences of mitochondrial proteins of the model organisms rep- resenting different phylogenetic groups. The amino acid sequences of the proteins were taken from the GenPept database. Table 1 presents the list of reference numbers of protein sequences from the organisms analyzed in the study. The protein sequences were analyzed with the use of the ProtParam program (6) to determine instability index values as in Guruprasad et al. (10). A protein whose instability index is lower than 40 is predicted as stable. A value above 40 predicts that the protein may be unstable (10).

\section{Results and discussion}

In the group of the 155 analyzed proteins, 38 exhibited instability (Tab. 2). The most unstable mitochondrial proteins were detected in Homo sapiens and Ambystoma mexicanum (5/13) as well as Drosophila melanogaster and Crocodylus porosus (4/13). The lowest number of unstable proteins was found in Caenorhabditis elegans (1/12), which is the most primitive organism in the analyzed group. Proteins ND1 (9/12) and ATP8 (8/12) were characterized by the highest instability of all the analyzed material. Proteins COX1 and COX3 did not exhibit instability in the studied group of organisms. The value of the instability index in the case of the COX3 proteins did not even exceed 30 . The highest values of the instability index were calculated for proteins ATP8, i.e. 68.59 in L. chalumnae, 67.92 in D. melanogaster, and 67.34 in $C$. porosis. The lowest value was noted in the case of protein ATP8 in G. gallus. For ATP8, the instability index had the most diverse values. Protein ND1 exhibited stability in the members of the invertebrate class (Tab. 2).

No studies to date have been conducted on the stability of all mitochondrial proteins in model organisms. The instability index value is calculated during in silico analysis of proteins (1); however, there are no examples of interpretation thereof in the literature or indication of its potential impact on cell physiol- 


\begin{tabular}{|c|c|c|c|c|}
\hline Gallus gallus & Mus musculus & $\begin{array}{c}\text { Canis lupus } \\
\text { familiaris }\end{array}$ & $\begin{array}{c}\text { Pan } \\
\text { tryglodytes }\end{array}$ & $\begin{array}{c}\text { Homo } \\
\text { sapiens }\end{array}$ \\
\hline NP_006920.1 & NP_904333.1 & NP_008476.1 & NP_008191.1 & YP_003024031 \\
\hline NP_006919.1 & NP_904332.1 & NP_008475.1 & NP_008190.1 & YP_003024030 \\
\hline NP_006917.1 & NP_904330.1 & NP_008473.4 & NP_008188.1 & YP_003024028.1 \\
\hline NP_006918.1 & NP_904331.1 & NP_008474.4 & NP_008189.1 & YP_003024029.1 \\
\hline NP_006921.2 & NP_904334.1 & NP_008477.4 & NP_008192.1 & YP_003024032.1 \\
\hline NP_006926.1 & NP_904340.1 & NP_008483.1 & NP_008198.1 & YP_003024038.1 \\
\hline NP_006915.1 & NP_904328.1 & NP_008471.1 & NP_008186.1 & YP_003024026.1 \\
\hline NP_006916.1 & NP_904329.1 & NP_008472.4 & NP_008187.1 & YP_003024027.1 \\
\hline NP_006922.1 & NP_904335.1 & NP_008478.4 & NP_008193.1 & YP_003024033.1 \\
\hline NP_006924.2 & NP_904337.1 & NP_008480.1 & NP_008195.1 & YP_003024035.1 \\
\hline NP_006923.1 & NP_904336.1 & NP_008479.1 & NP_008194.1 & YP_003024034.1 \\
\hline NP_006925.1 & NP_904338.1 & NP_008481.4 & NP_008196.1 & YP_003024036.1 \\
\hline NP_006927.1 & NP_904339.1 & NP_008482.4 & NP_008197.1 & YP_003024037.1 \\
\hline
\end{tabular}

of unstable amino acids increasing the instability index, this is sufficient to cause protein instability. It is worth emphasizing in this study that there is at least one unstable protein among proteins encoded in the mitochondrial genome in each of the analyzed organisms (Tab. 2). The phenomenon of respiratory chain protein instability may thus have a strategic function, although it seems to be disadvantageous to the stability of the protein structure perse.

Mitochondrial generation of reactive oxygen species (ROS) is an inevitable consequence of oxidative ATP production and the primary cause of macromolecular damage. Some damage is not repaired, thus

ogy. Research is more often focused on other factors affecting the stability of the protein structure, e.g. hydrophobic interactions and hydrogen bonds (16). It has also been shown that protein stability can be associated with stabilization centers (SCs), which exert an effect on maintenance of the tertiary structure. Stabilization centers are associated with the fact that the functional sites of proteins are usually composed of only a couple of residues (13). As suggested by Magyar et al. (13), there might be a few additional residues responsible for the stability of the three-dimensional structure of proteins. If stabilization centers are composed of a pair causing progressive failure of cellular machinery, ageing-related diseases, and ageing. As demonstrated by Muller et al. (15), mitochondrial ROS convincingly determines the lifespan in Caenorhabditis elegans. In their research, Senoo-Matsuda et al. (18) indicated that point mutations in complex I and complex II of the mitochondrial electron transfer chain enhance superoxide production and reduce the lifespan of C. elegans. When both complexes are mutated, the phenotype is lethal (18). Point mutations in an unstable protein may lead to a change from one dipeptide with a high positive value to another with a negative value, consequently

Tab. 2. Values of the instability index of mitochondrial proteins in the analyzed organisms

\begin{tabular}{|c|c|c|c|c|c|c|c|c|c|c|c|c|c|}
\hline Protein & 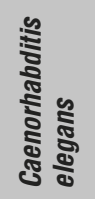 & 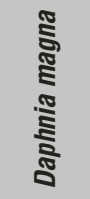 & 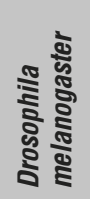 & 离 & 옳 & 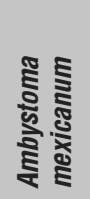 & 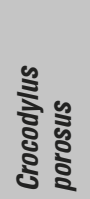 & 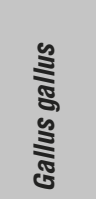 & 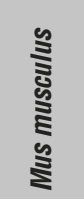 & 言: & $\frac{\oiiint}{\frac{5}{5}}$ & 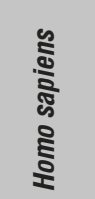 & $B^{* *}$ \\
\hline ATP6 & 33.05 & 34.22 & 40.85 & 40.08 & 34.83 & 38.03 & 42.67 & 47.11 & 31.88 & 32.34 & 32.19 & 34.74 & 4 \\
\hline ATP8 & N/D & 52.49 & 67.92 & 68.59 & 22.33 & 49.80 & 67.34 & 13.66 & 45.38 & 36.04 & 53.61 & 51.40 & 8 \\
\hline $\operatorname{cox} 1$ & 30.45 & 29.95 & 27.86 & 24.97 & 26.13 & 31.51 & 34.00 & 29.60 & 25.43 & 27.96 & 27.55 & 28.97 & 0 \\
\hline $\operatorname{cox} 2$ & 21.61 & 33.30 & 38.78 & 47.92 & 45.91 & 37.21 & 37.53 & 35.24 & 36.18 & 36.64 & 35.05 & 37.11 & 2 \\
\hline $\operatorname{coX} 3$ & 26.92 & 22.82 & 29.44 & 26.50 & 27.09 & 20.86 & 26.39 & 28.13 & 24.78 & 19.67 & 24.04 & 22.96 & 0 \\
\hline СYTB & 32.81 & 35.74 & 32.98 & 28.51 & 33.31 & 42.83 & 36.05 & 36.30 & 39.01 & 38.39 & 39.55 & 41.02 & 1 \\
\hline ND1 & 31.09 & 31.93 & 35.78 & 41.87 & 45.72 & 40.43 & 42.15 & 45.82 & 46.57 & 46.15 & 41.70 & 41.94 & 9 \\
\hline ND2 & 29.98 & 41.06 & 33.66 & 35.85 & 38.54 & 44.82 & 30.82 & 34.04 & 31.71 & 29.67 & 37.46 & 34.35 & 2 \\
\hline ND3 & 22.77 & 37.48 & 43.98 & 30.70 & 29.33 & 27.51 & 31.36 & 20.12 & 34.16 & 30.24 & 47.86 & 50.62 & 2 \\
\hline ND4 & 37.62 & 27.90 & 35.51 & 32.92 & 36.32 & 42.49 & 39.20 & 37.56 & 38.19 & 37.22 & 32.63 & 35.98 & 1 \\
\hline ND4L & 13.87 & 23.68 & 21.11 & 32.96 & 28.34 & 39.57 & 41.97 & 41.46 & 42.79 & 43.22 & 46.00 & 46.00 & 6 \\
\hline ND5 & 27.85 & 35.65 & 40.12 & 35.82 & 28.01 & 38.80 & 32.79 & 30.33 & 32.33 & 31.51 & 32.10 & 33.95 & 1 \\
\hline ND6 & 43.70 & 34.61 & 18.30 & 43.63 & 21.71 & 29.63 & 15.90 & 15.39 & 29.63 & 22.18 & 28.44 & 29.48 & 2 \\
\hline$A^{*}$ & 1 & 2 & 4 & 4 & 2 & 5 & 4 & 3 & 3 & 2 & 3 & 5 & 38 \\
\hline
\end{tabular}

Explanations: Values marked in green indicate protein instability; $A^{*}$ - number of unstable proteins in each organism; $\mathrm{B}^{* *}-$ number of unstable proteins in the analyzed organisms 
making the protein stable (10). It is quite possible that point mutations in the genes of complex I (Tab. 2) cause an increase in the instability index of protein ND6 and have a negative impact on the lifespan of C. elegans. Nematodes are also more tolerant to anaerobic conditions and more resistant to oxidative stress than mammals (15).

The free radical mechanism may also induce nonsynonymous mutations affecting the amino acid sequence in the mitochondrial proteins in other model organisms, which may result in an increase in the instability index value. As shown by Kowal et al. (11) in a dog with a mammary tumor, in the case of the amino acid substitution in protein ND4L, as a result of mutation in the ND4L gene sequence, the instability index value indicated a transition in the stability of the protein from unstable in the reference sequence to stable in the blood and tumor cells of the dog. The difference in one position of the amino acid sequence had a positive effect on protein stability, i.e. the change transforms an unstable protein before the mutation into a stable one after the mutation (11). This can be verified by the theory proposed by Guruprasad et al. (10), who argue that protein instability is caused by the presence of certain dipeptides in the protein structure. The literature also provides reports on human (8) and canine (21) tumors, in which, due to mutations, physiologically unstable proteins (ATP8 and ND1, respectively) remain unstable, can have a negative impact on the function of proteins, and contribute to disorders in electron transport. There are also reports of cases where somatic mutations associated with canine tumors resulting in amino acid changes in proteins COX2 and COX3 did not alter protein stability (23).

It is difficult to assess unequivocally the impact of the instability of mitochondrial proteins on the function of higher organisms. Since the number of unstable proteins varies (Tab. 2), likewise the living environment of higher organisms, the relationship between protein instability and oxidative stress cannot be demonstrated clearly. However, it is worth emphasizing that instability is more often detected in smaller subunits such as ATP8 or ND4L than in larger molecules, with the exception of ND1. This can be explained by the fact that the structure of smaller proteins is more sensitive to destabilization than that of larger proteins.

In the case of $D$. melanogaster, oxygen tension can modulate the lifespan, but it is inconclusive in mice and humans (15). Experiments conducted by Baret et al. (3) have shown a quasi-linear inverse relationship between the lifespan and oxygen tension in Drosophila. Over-expression of antioxidant defenses in mice does not generally increase the lifespan (17). Currently, seven separate sites of mammalian mitochondrial ROS production have been identified and widely accepted. The sites with the greatest maximum capacities to produce ROS are located in complex I (site IQ) and complex III (site IIIQo), although the other five sites also have significant maximum rates (4). Complex I produces superoxide to the matrix, whereas complex III produces it to the matrix and intermembrane space at about equal rates in under de-energized conditions (4). Probably overproduction of free oxygen radicals in complex I results from the instability of proteins forming the complex in some organisms. This observation is especially important given the physiological functions of free radicals in higher organisms. The instability of mitochondrial proteins such as ND1 and ND4L (Tab. 2) in amphibians, reptiles, birds, and mammals can therefore be crucial for the generation of free radicals supporting physiological processes. In turn, the absence of protein COX instability (Tab. 2) can be subject to evolutionary pressure protecting against overproduction of free radicals, which could enter the cytoplasm from the intermembrane space, thus damaging protein and nuclear DNA. It is therefore probable that the instability of the specific mitochondrial proteins in some organisms is associated with the production of free oxygen radicals. Proteins COX2 are only unstable in fish L. chalumnae and D. rerio (Tab. 2), which may be associated with the specific phylogenetic function of free radicals.

Currently, it is irrefutable that the proper function of living organisms requires the presence of free radicals at a certain relatively low level and their excess needs to be counteracted (7). ROS are involved in many metabolic reactions: inactivation of viruses and bacteria, regulation of cell growth, inter- and intracellular signaling, synthesis of tissue hormones in the form of prostacyclins, thromboxanes, and leukotrienes from polyunsaturated fatty acids, and activation of cytochrome P450 and many genes in higher organisms $(4,9)$. Therefore, the higher number of unstable mitochondrial proteins in vertebrates (Tab. 2) may result from the need to produce free oxygen radicals to ensure their proper physiological concentration.

To sum up, the results of this pioneering research indicate the presence of at least one unstable mitochondrial protein in each of the studied organisms. The type and number of unstable mitochondrial proteins is species specific. Protein instability in lower organisms may be associated with resistance to oxidative stress. In turn, this phenomenon in higher organisms may be related to the physiological generation of free oxygen radicals, which serve multiple functions in metabolic processes. Research needs to be continued to identify the causes of protein instability.

\section{References}

1.Al-Banna L., Sadder M. T., Lafi H. A., Dawabah A. A., Al-Nadhari S. N. Bioinformatics analysis of ubiquitin expression protein gene from Heterodera latipons. Saudi J. Biol. Sci. 2018, doi.org/10.1016/j.sjbs.2018.06.005.

2. Bai Y., Sharma L. K., Lu J.: Implications of mitochondrial DNA mutations and mitochondrial dysfunction in tumorigenesis. Cell Res. 2009, 19, 802-815.

3. Baret P., Fouarge A., Bullens P., Lints F. A.: Life-span of Drosophila melanogaster in highly oxygenated atmospheres. Mech. Ageing Dev. 1994, 76, 25-31. 4. Brand M. D.: The sites and topology of mitochondrial superoxide production. Exp. Gerontol. 2010, 45, 466-472. 
5. Cogliati S., Lorenzi I., Rigoni G., Caicci F., Soriano M. E.: Regulation of Mitochondrial Electron Transport Chain Assembly. J. Mol. Biol. 2018, 430, 4849-4873.

6. Gasteiger E., Hoogland C., Gattiker A., Duvaud S., Wilkins M. R., Appel R. D. Bairoch A.: Protein Identification and Analysis Tools on the ExPASy Server, [in:] John M. Walker (ed): The Proteomics Protocols Handbook. Humana Press 2005, p. 571-607.

7. Gebicki J. M., Bartosz G.: The role of proteins in propagation of damage induced by reactive oxygen species in vivo. Postepy Biochem. 2010, 56, 115-123.

8. Grzybowska-Szatkowska L., Slaska B., Rzymowska J., Brzozowska A., Floriańczyk B.: Novel mitochondrial mutations in the ATP6 and ATP8 genes in patients with breast cancer. Mol. Med. Rep. 2014, 4, 1772-1778.

9. Grzybowska-Szatkowska L., Slaska B.: Mitochondrial DNA and carcinogenesis (Review). Mol. Med. Rep. 2012, 6, 923-930.

10. Guruprasad K., Reddy B. B., Pandit M. W.: Correlation between stability of a protein and its dipeptide composition: a novel approach for predicting in vivo stability of a protein from its primary sequence. Protein Eng. Des. Sel. 1990, 4, 155-161.

11. Kowal K., Ślaska B., Bownik A., Horecka B., Gawor J., Śmiech A., TkaczykA. Analysis of mitochondrial genome from Labrador (Canis lupus familiaris) with mammary gland tumour reveals novel mutations and polymorphisms. Ann. Anim. Sci. 2019 (in press).

12. Kussmaul L., Hirst J.: The mechanism of superoxide production by NADH: ubiquinone oxidoreductase (complex I) from bovine heart mitochondria. P. Natl. Acad. Sci. USA 2006, 103, 7607-7612.

13. Magyar C., Gromiha M., Savoly I.: The role of stabilization centers in protein thermal stability. Biochem. Bioph. Res. Co. 2016 471, 57-62.

14. Mohanty K., Dada R., Dada T.: Neurodegenerative eye disorders: role of mitochondrial dynamics and genomics. Asia Pac. J. Ophthalmol. 2016, 5, 293-299.
15. Muller F. L., Lustgarten M. S., Jang Y., Richardson A., Van Remmen H.: Trendsin oxidative aging theories. Free Radical Biol. Med. 2007, 43, 477-503.

16. Pace C. N., Scholtz J. M., Grimsley G. R.: Forces stabilizing proteins FEBS Lett. 2014, 588, 2177-2184.

17. Perez V. I., Van Remmen H., Bokov A., Epstein C. J., Vijg J., Richardson A.: The overexpression of major antioxidant enzymes does not extend the lifespan of mice. Aging Cell 2009, 8, 73-75.

18. Senoo-Matsuda N., Yasuda K., Tsuda M., Ohkubo T., Yoshimura S., Nakazawa H., Hartman P. S., Ishii N.: A defect in the cytochrome b large subunit in complex II causes both superoxide anion overproduction and abnormal energy metabolism in Caenorhabditis elegans. J. Biol. Chem. 2001, 276, 41553-41558.

19. Sharma L. K., Lu J., Bai Y.: Mitochondrial respiratory complex I: structure, function and implication in human diseases. Curr. Med. Chem. 2009, 16, 1266-1277.

20.Singh K. K., Russell J., Sigala B., Zhang Y., Williams J., Keshav K. F.: Mitochondrial DNA determines the cellular response to cancer therapeutic agents. Oncogene 1999, 18, 6641-6646.

21. Slaska B., Grzybowska-Szatkowska L., Nisztuk S., Surdyka M., Rozanska D.: Mitochondrial DNA polymorphism in genes encoding ND1, COI and CYTB in canine malignant cancers. Mitochondr. DNA 2015, 26, 452-458.

22. St-Pierre J., Buckingham J. A., Roebuck S. J., Brand M. D.: Topology of superoxide production from different sites in the mitochondrial electron transport chain. J. Biol. Chem. 2002, 277, 44784-44790.

23. Surdyka M., Slaska B.: Defect in ND2, COX2, ATP6, and COX3 mitochondrial genes as a risk factor for canine mammary tumour. Vet. Comp. Oncol. 2017, $15,1062-1072$.

Corresponding author: Prof. dr hab. Brygida Ślaska, DVM, PhD, Akademicka 13, 20-950 Lublin, Poland; e-mail: brygida.slaska@up.lublin.pl 